\title{
Effect of chirp on self-modulation and laser wakefield electron acceleration in the regime of quasimonoenergetic electron beam generation
}

\author{
B. S. Rao, ${ }^{*}$ A. Moorti, P. A. Naik, and P. D. Gupta \\ Laser Plasma Division, Raja Ramanna Centre for Advanced Technology, Indore 452 013, India
}

(Received 9 May 2013; published 25 September 2013)

\begin{abstract}
Strongly self-modulated regime of laser wakefield electron acceleration has been experimentally studied using positively/negatively chirped Ti:sapphire laser pulses with duration $\geq 45 \mathrm{fs}$, and intensity $\leq 1.2 \times 10^{18} \mathrm{~W} / \mathrm{cm}^{2}$ interacting with a plasma having density $>5 \times 10^{19} \mathrm{~cm}^{-3}$. The total charge of the high energy electrons produced from self-injection and acceleration in the wakefield was found to depend strongly and asymmetrically on the magnitude and sign of the chirp, which was also corroborated by the forward Raman scattering signals. Generation of low divergence ( $\leq 10 \mathrm{mrad})$, quasimonoenergetic electron beam was observed with a maximum energy of $\sim 28 \mathrm{MeV}$ for a positively chirped laser pulse of duration $\sim 70 \mathrm{fs}$. The observations are explained considering faster rise time associated with a positive chirped laser pulse which generates intense seed for stronger modulation of the laser pulse and consequently stronger wakefield excitation leading to higher electron beam charge and energy.
\end{abstract}

\section{INTRODUCTION}

The laser wakefield acceleration (LWFA) [1] scheme utilizes large amplitude plasma waves for accelerating electrons to relativistic energies over extremely short distances. Therefore, this scheme is a promising one as a new technology, which could lead to downsizing of future high energy accelerators. The field has progressed rapidly over the past decade [2], through several breakthrough experiments which demonstrated the generation of low divergence $(<10 \mathrm{mrad})$, quasimonoenergetic $(\Delta E / E<$ $10 \%$ ), and ultrashort ( $<10 \mathrm{fs}$ ) bunches of relativistic electrons with energies up to $1 \mathrm{GeV}$ in the "bubble regime" which requires laser pulse length $c \tau \sim \lambda_{p} / 2$ [3-10]. In order to control the accelerated electron beam parameters in this regime, various schemes have been investigated and controlled injection has been successfully demonstrated [11-15].

Quasimonoenergetic electron bunches have also been produced in the strongly self-modulated LWFA (SM-LWFA) regime by using longer pulses $\left(c \tau \gg \lambda_{p}\right)$ and higher plasma density [16-18]. In this regime, a single long laser pulse with duration $c \tau \gg \lambda_{p}$ breaks up into multiple short pulses through forward Raman scattering (FRS) instability [19], each of which has a width of the order of the plasma wavelength $\lambda_{p}$ [16]. A large amplitude wakefield is associated with the breakup of the long pulse and the formation of the pulse train. The field of this longitudinal wave is sufficiently large to self-trap electrons

\footnotetext{
*sunnyb@ rrcat.gov.in
}

Published by the American Physical Society under the terms of the Creative Commons Attribution 3.0 License. Further distribution of this work must maintain attribution to the author(s) and the published article's title, journal citation, and DOI. from the background plasma, and accelerate them to high energies. In addition to the pulse length criterion, the pulse power $P$ should also exceed the critical power for relativistic self-focusing, $P_{c}(\mathrm{GW})=16.2\left(n_{c} / n_{e}\right)$, where $n_{e}$ is the plasma electron density, and $n_{c}$ is the critical density [20]. Since $\lambda_{p} \propto n_{e}{ }^{-1 / 2}$ and $P_{c} \propto n_{e}{ }^{-1}$ for typical laser parameters used in LWFA experiments, the conditions for SMLWFA are satisfied at a sufficiently high plasma density $>10^{19} \mathrm{~cm}^{-3}$. This regime is interesting for high charge electron beam generation with a few tens of $\mathrm{MeV}$ energy using few TW power laser systems.

FRS is an inherent and important physical process occurring in the SM-LWFA. Therefore, controlling the FRS and the wakefields is essential for controlling the charge, energy, and quality of the electron beam in this regime. It has been suggested that by introducing a chirp in the laser pulse, the growth of FRS can be controlled [21-23] along with the wakefield amplitude and the electron beam parameters in the laser wakefield electron acceleration [24-27]. However, there are only a few experimental studies on the effect of frequency chirped laser pulses on electron acceleration. The effect of laser pulse chirp was first studied in a SM-LWFA experiment by Leemans et al. [28], where they observed enhanced FRS and higher electron yield with a positive chirp laser pulse. Recently, Hafz et al. [29] have reported generation of relativistic electron beams with stable pointing angle from a weakly nonlinear laser wakefield acceleration regime, using positive chirped laser pulses. In both these experiments, the effect of chirp on the electron beam energy was not studied.

In this paper, we present an experimental study on the effect of laser chirp on FRS driven laser self-modulation and laser wakefield acceleration using positively/negatively chirped Ti:sapphire laser pulses with duration $\geq 45 \mathrm{fs}$, interacting with high density $\left(>5 \times 10^{19} \mathrm{~cm}^{-3}\right)$ plasma. 
We have observed asymmetry in the electron charge with respect to (wrt) the chirp. The asymmetry was also found to depend on the plasma density (larger asymmetry for lower plasma density). The strength of the FRS signal was also found to depend on the sign of the laser pulse chirp. The use of positively chirped laser pulse of about 70-80 fs duration resulted in enhanced FRS with nearly twofold enhancement in the accelerated electron charge, at a plasma density of $6.5 \times 10^{19} \mathrm{~cm}^{-3}$. At a higher plasma density of $8.5 \times$ $10^{19} \mathrm{~cm}^{-3}$, generation of low divergence quasimonoenergetic electron beam was also observed, indicating strong self-modulation of the laser pulse leading to electron acceleration in the self-modulated bubble regime. The observations are explained by considering laser pulse shape asymmetry (fast rise time for positively chirped pulse) which arises due to uncompensated higher order phase nonlinearity in the laser pulse. This method is found to be suitable for controlling the self-modulation and consequently the charge and the quality of the electron beam in the SM-LWFA regime.

\section{EXPERIMENTAL SETUP}

A schematic of the experimental setup is shown in Fig. 1. The experiment was performed using the chirped pulse amplification (CPA) based Ti:sapphire laser system at Laser Plasma Division of Raja Ramanna Centre for Advanced Technology (RRCAT), Indore, India. In this laser system, femtosecond duration laser pulses of about $1 \mathrm{~nJ}$ energy from oscillator were first temporally stretched to about $300 \mathrm{ps}$ duration, amplified to about $0.6 \mathrm{~J}$ level, and finally compressed to a pulse of full width at half maximum (FWHM) duration of 45 fs using an optical compressor consisting of a pair of parallel gratings (groove density $1500 / \mathrm{mm}$, angle of incidence of $45^{\circ}$ ) separated by an optimal distance $(365 \mathrm{~mm})$ in vacuum. The laser beam was transported from the compressor to the plasma chamber ( $3.5 \mathrm{~m}$ distance) through an evacuated beam transport line. The optimal grating separation in the compressor that produces the minimum pulse duration at the interaction point is referred to as the "compressor zero" which was determined as per the following procedure. The laser beam was reflected out of the evacuated beam transport line through a glass window and was sent to a single shot

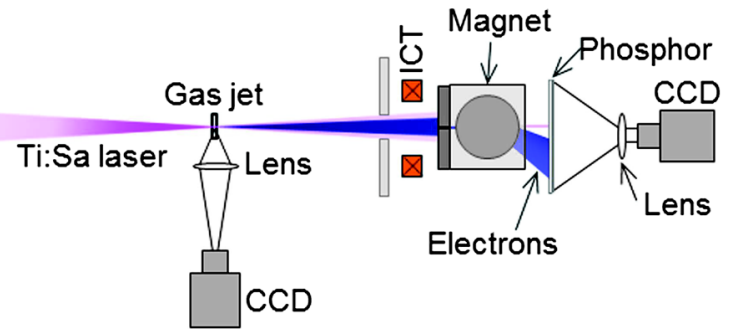

FIG. 1. A schematic diagram of the experimental setup for electron acceleration. second order autocorrelator (located in air at a distance of $1 \mathrm{~m}$ from the glass window) for the pulse duration measurement. The grating separation was varied to obtain the minimum laser pulse duration. However, the group velocity dispersion in the glass window introduces a positive chirp (measured to be $\sim 10 \mathrm{fs}$ ) in the laser pulse (the contribution of air being negligibly small). To account for this extra chirp of the glass window, a positive chirp of $\sim 10$ fs was introduced in the compressor to get the minimum pulse duration at the plasma chamber (i.e. without glass window). This position of the gratings was taken as the compressor zero. The laser pulse duration and consequently the chirp characteristics were varied by scanning the grating pair separation from its optimal separation. The laser pulses of duration $\tau \geq 45 \mathrm{fs}$ after the compressor have central wavelength $\lambda_{0} \simeq 800 \mathrm{~nm}$, FWHM spectral width $\Delta \lambda \simeq 20 \mathrm{~nm}\left(\Delta \lambda / \lambda_{0}=2.5 \%\right)$, and maximum peak power of $9 \mathrm{TW}$. The laser beam after the pulse compressor was focused using an $f / 10$ gold-coated offaxis parabolic mirror to a spot of FWHM diameter $d_{0}=$ $18 \mu \mathrm{m}$ at the entrance edge of a $1.2 \mathrm{~mm}$ wide rectangular shaped (size: $10 \mathrm{~mm} \times 1.2 \mathrm{~mm}$ ) well characterized supersonic helium gas jet. The Rayleigh range of the focused laser beam was $Z_{R}=\pi d_{0}^{2} /\left(2 M^{2} \lambda \ln 2\right) \simeq 300 \mu \mathrm{m}$ and the peak intensity at the waist was $I_{L}=4 P_{0} \ln 2 / \pi d_{0}^{2} \simeq$ $1.2 \times 10^{18} \mathrm{~W} / \mathrm{cm}^{2}\left(a_{0} \simeq 0.75\right)$, considering about $50 \%$ $(4.5 \mathrm{TW})$ of the total power contained in the focal spot. The interaction chamber was evacuated to a pressure of $\sim 10^{-5}$ mbar. The backing pressure of the gas jet was varied to provide plasma electron density $\left(n_{e}\right)$ in the range of $1 \times 10^{19}-1 \times 10^{20} \mathrm{~cm}^{-3}$.

An integrating current transformer (Bergoz: ICT-082070-5:1) was used to measure the energy integrated accelerated electron beam charge. A thick aluminum disk with a central hole was kept behind the gas jet to allow electrons emitted within a full cone angle of $\leq 20^{\circ}$ to pass through the ICT. A $\operatorname{Gd}_{2} \mathrm{O}_{2} \mathrm{~S}: \mathrm{Tb}$ phosphor screen (DRZ-high), coupled with a 12-bit CCD camera for imaging, was used for online measurement of the accelerated electron beam spatial profile. The energy spectrum of the electrons was measured with a single shot electron spectrograph consisting of a dipole magnet having two circular permanent magnets of $50 \mathrm{~mm}$ diameter, with $9 \mathrm{~mm}$ pole gap, to deflect the electrons which are detected on the phosphor screen. The magnetic field profile between the pole pieces had a flattop with effective magnetic field $B_{\text {eff }}=0.46 \mathrm{~T}$. A rectangular slit $(5 \mathrm{~mm} \times 9 \mathrm{~mm})$, made in a combination of $12 \mathrm{~mm}$ thick aluminum and $6 \mathrm{~mm}$ thick lead, was placed at the entrance of the magnet.

The laser-plasma interaction was observed through imaging of the incoherent Thomson side scattering of the laser radiation, with $5 \times$ magnification, on to a 12-bit CCD. The laser polarization direction was horizontal, perpendicular to the observation angle of the side scattering monitor. A narrow band-pass filter was placed in front of 
the CCD to allow scattered radiation having wavelength within $800 \pm 20 \mathrm{~nm}$. A part of the transmitted laser light from the interaction region, at an angle of $6^{\circ}$ wrt laser axis, was attenuated and reflected using a glass wedge, and collected by a lens which focused it on to the entrance slit of an optical spectrograph covering spectral range from $550-1100 \mathrm{~nm}$, to detect and measure the wavelength shifts in the transmitted spectrum due to forward Raman scattering. A long pass filter (RG 850), in combination with an appropriate number of neutral density filters, was placed in front of the spectrometer to significantly attenuate the transmitted laser spectrum below $850 \mathrm{~nm}$. This improves the sensitivity of detection of the less intense redshifted signal expected due to stimulated FRS.

\section{RESULTS AND DISCUSSION}

For a chirped laser pulse, one can define the instantaneous frequency $\omega$ as $\omega=\omega_{0}+b t$, where $t$ is the time in the pulse reference frame, $b$ is the chirp parameter given by $b=2 \ln 2 / \tau^{2}\left[\left(\tau / \tau_{o}\right)^{2}-1\right]^{1 / 2}$, where $\tau$ and $\tau_{o}$ are the FWHM duration of the chirped and unchirped pulse, respectively. Hence, for a negative chirp $(b>0)$, the "blue" frequencies are located at the front of the pulse, and for a positive chirp $(b<0)$, the "red" frequencies are located at the front of the pulse. Positively/negatively chirped pulses, with duration ranging from 45 to \pm 450 fs (corresponding intensities varied from $1.2 \times 10^{18} \mathrm{~W} / \mathrm{cm}^{2}$ down to $1.2 \times 10^{17} \mathrm{~W} / \mathrm{cm}^{2}$ ), were generated by translating one of the two gratings of the pulse compressor by 0 to $\pm 2 \mathrm{~mm}$ from their optimal separation for minimum pulse duration. Hereafter, "plus" or "minus" signs will be used (except for $45 \mathrm{fs}$ ) together with the pulse duration to indicate positively or negatively chirped laser pulses.

First, the electron beam generation for the unchirped laser pulse of 45 fs duration was studied. Self-trapped and accelerated electrons were detected for plasma density $\geq 5 \times 10^{19} \mathrm{~cm}^{-3}$ and the charge of the accelerated electrons was observed to increase exponentially with the plasma density. Further, generation of low divergence, quasimonoenergetic electron beam was observed for a plasma density of $\simeq 8.5 \pm 0.5 \times 10^{19} \mathrm{~cm}^{-3}$ [17]. Next, the effect of chirp on self-modulation and electron acceleration for two different plasma densities, $n_{e} \simeq 6.5 \pm$ $0.5 \times 10^{19} \mathrm{~cm}^{-3}$ (case 1) and $n_{e} \simeq 8.5 \pm 0.5 \times 10^{19} \mathrm{~cm}^{-3}$ (case 2), was studied. Figure 2 shows the variation of normalized charge of the electrons with relative separation of compressor gratings, for case 1 and case 2. For easy reference, the figure also shows the variation of the laser pulse duration with the grating separation. In both cases, normalization of the charge was done wrt the total charge observed with the unchirped pulse (45 fs) interaction. The total charge for compressor zero setting was 2 and $8 \mathrm{nC}$, respectively, for plasma density $6.5 \times 10^{19} \mathrm{~cm}^{-3}$ and $8.5 \times 10^{19} \mathrm{~cm}^{-3}$. An asymmetry in the electron beam charge wrt the laser pulse chirp was observed for both

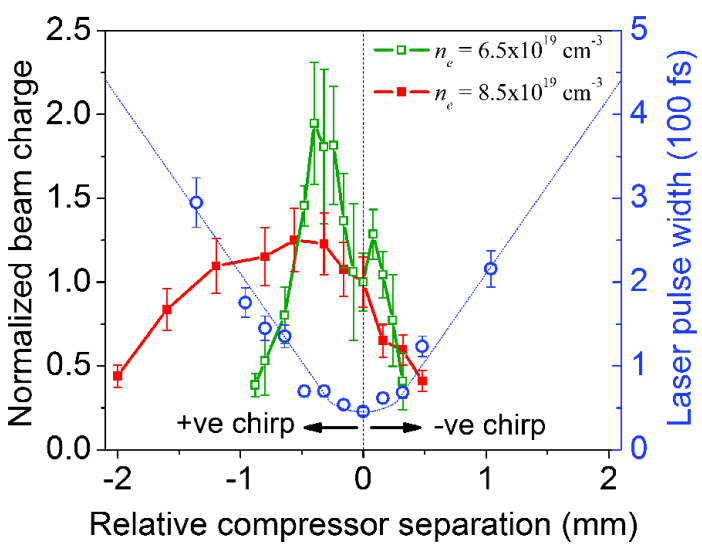

FIG. 2. Variation in the integrated electron beam charge at two different plasma densities (solid squares: $8.5 \times 10^{19} \mathrm{~cm}^{-3}$; and hollow squares: $6.5 \times 10^{19} \mathrm{~cm}^{-3}$ ) with compressor grating pair separation (chirp) measured with respect to the "zero" setting (separation corresponding to minimum pulse duration). The total charge for zero setting of the compressor is 2 and $8 \mathrm{nC}$, respectively, for plasma density $6.5 \times 10^{19} \mathrm{~cm}^{-3}$ and $8.5 \times$ $10^{19} \mathrm{~cm}^{-3}$. The variation of the laser pulse width (circles) with grating separation is also shown.

the plasma densities. The maximum charge was not observed for the shortest ( $45 \mathrm{fs}$ ) laser pulse but for longer laser pulses with a small amount of positive chirp. The charge increased by nearly $100 \%$ and $25 \%$ for $n_{e} \simeq 6.5 \pm$ $0.5 \times 10^{19} \mathrm{~cm}^{-3}$ and $8.5 \pm 0.5 \times 10^{19} \mathrm{~cm}^{-3}$ respectively, when positively chirped pulses of about +80 to +85 fs duration were used. The minimum laser power at which electrons were observed was about $1.1 \mathrm{TW}$, corresponding to positively chirped laser pulse of +185 fs duration for $n_{e} \simeq 6.5 \pm 0.5 \times 10^{19} \mathrm{~cm}^{-3}$ and $0.45 \mathrm{TW}$ corresponding to $+420 \mathrm{fs}$ for $8.5 \pm 0.5 \times 10^{19} \mathrm{~cm}^{-3}$. This power is higher than the critical power $P_{c} \simeq 0.43 \mathrm{TW}$ and $0.33 \mathrm{TW}$ required for relativistic self-focusing at the two densities. Along with the accelerated electrons, laser self-focusing channels of length $400-500 \mu \mathrm{m}$ were also observed in the Thomson scattering side images.

Figure 3 shows the measured spectra of transmitted laser light wrt the laser chirp/grating separation for $n_{e} \simeq$ $6.5 \pm 0.5 \times 10^{19} \mathrm{~cm}^{-3}$ and $8.5 \pm 0.5 \times 10^{19} \mathrm{~cm}^{-3}$. The sidebands at longer wavelengths due to stimulated FRS can be clearly seen. The wavelength shift of the sideband with change in plasma density is consistent with FRS. The variation in the amplitude of the FRS signal with laser pulse chirp for a fixed density was consistent with the observed asymmetry in the electron beam charge with chirp. This clearly suggests that the laser pulse was undergoing strong self-modulation through stimulated FRS and resulting in excitation of large amplitude wakefields which could self-trap and accelerate background plasma electrons to high energy. The transmitted spectra also show redshifting of the laser light which is probably due to laser photon deceleration in the wakefield [30]. Since a high pass filter (RG-850), which strongly attenuates the 


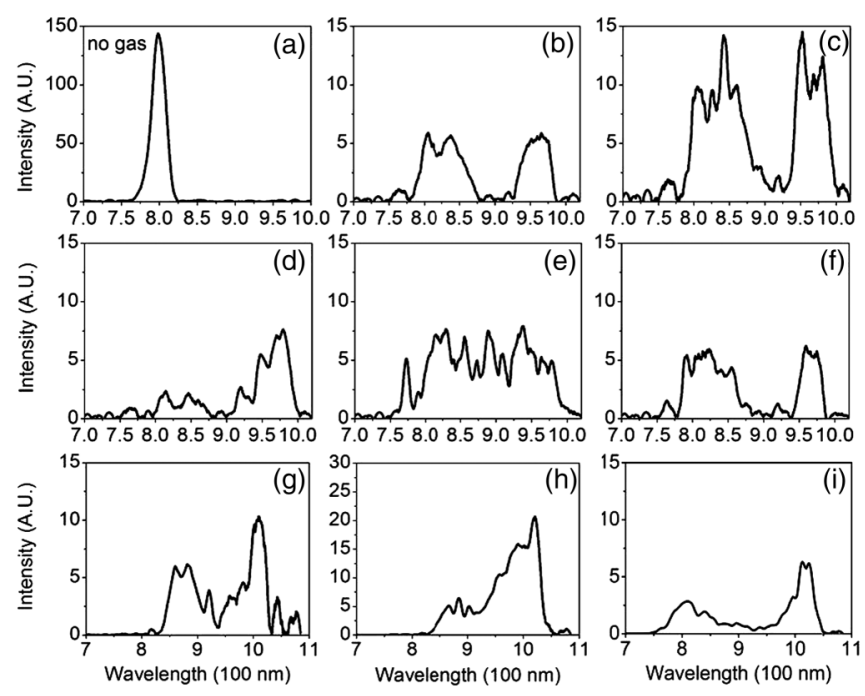

FIG. 3. The transmitted laser spectra at two different plasma densities: (a) without interaction with plasma, (b) to (f) with interaction at $n_{e} \simeq 6.5 \pm 0.5 \times 10^{19} \mathrm{~cm}^{-3}$, for grating separation of 0 (45 fs), $-320 \mu \mathrm{m}$ (70 fs), $-600 \mu \mathrm{m}$ (125 fs), $-800 \mu \mathrm{m}$ (170 fs), and $+200 \mu \mathrm{m}$ (52 fs), respectively, (g) to (i) at $n_{e} \simeq 8.5 \pm 0.5 \times 10^{19} \mathrm{~cm}^{-3}$ for grating separation of 0 (45 fs), $-400 \mu \mathrm{m}$ ( $85 \mathrm{fs}$ ), and $+200 \mu \mathrm{m}$ (52 fs), respectively. No distinct Raman peak could be resolved from the transmitted spectra due to large broadening of laser spectrum [as shown in (e)] for grating separation $\gtrsim-800 \mu \mathrm{m}$ for a density of $6.5 \pm$ $0.5 \times 10^{19} \mathrm{~cm}^{-3}$. Similar observation was made for $n_{e} \simeq 8.5 \pm$ $0.5 \times 10^{19} \mathrm{~cm}^{-3}$ at grating separation of $>-400 \mu \mathrm{m}$ (spectra not shown here). Raman peaks disappear for grating separation $\gtrsim+200 \mu \mathrm{m}$ (-ve chirp).

spectrum below $850 \mathrm{~nm}$, was used to attenuate the transmitted laser light, the blueshifted anti-Stokes satellite band due to FRS and blueshift due to photon acceleration [30] could not be seen in Fig. 3.

With the shortest (45 fs) laser pulse interaction, the electron beam profile measurements for $n_{e} \geq 5 \times$ $10^{19} \mathrm{~cm}^{-3}$ showed usually large divergence $(\geq 100 \mathrm{mrad})$ electron beams except at $n_{e} \simeq 8.5 \pm 0.5 \times 10^{19} \mathrm{~cm}^{-3}$, where low divergence ( $\leq 10 \mathrm{mrad}$ ) electron beam was produced, as shown in Fig. 4(a), with highest probability. At this density, the low divergence electron beam was present even when positive chirp was introduced up to about $+250 \mathrm{fs}$, while it vanished on introducing a small amount of negative chirp ( $\geq-60 \mathrm{fs}$ ). In the case of $n_{e} \simeq$ $6.5 \pm 0.5 \times 10^{19} \mathrm{~cm}^{-3}$, once in a while, a low divergence electron beam was observed, as shown in Fig. 4(b), when positive chirp around $85 \mathrm{fs}$ was introduced. The figure shows one intense low divergence electron beam along with two more less intense electron beams, which can be attributed to multiple injection. The energy spectrum of the electron beam was measured at a plasma density of $n_{e} \simeq$ $8.5 \pm 0.5 \times 10^{19} \mathrm{~cm}^{-3}$ for different positive chirp values leading to variation in the laser pulse duration from 45 to 135 fs. Typical recorded spectra are shown in Fig. 5.
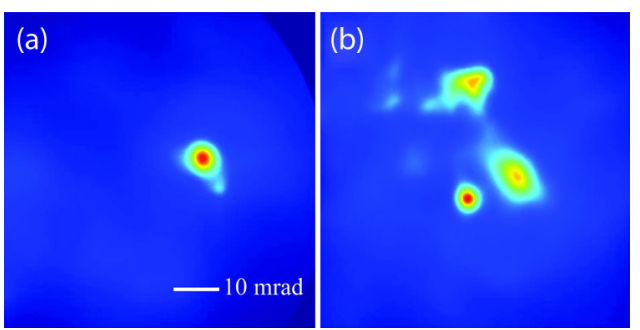

FIG. 4. (a) The electron beam profile at $n_{e} \simeq 8.5 \pm 0.5 \times$ $10^{19} \mathrm{~cm}^{-3}$, for a grating separation of $0 \mu \mathrm{m}$ (45 fs). (b) Image of multiple low divergence $(\$ 10 \mathrm{mrad})$ and high energy $\left(\gtrsim 2.5 \mathrm{MeV}\right.$ ) electron beams at $n_{e} \simeq 6.5 \pm 0.5 \times 10^{19} \mathrm{~cm}^{-3}$, for a grating separation of $-400 \mu \mathrm{m}$ (85 fs).

Generation of collimated, quasimonoenergetic electron beams was observed for the above range of positive chirped laser pulse duration, with maximum electron beam energy of $28 \mathrm{MeV}$ observed for a positive chirped laser pulse of $70 \mathrm{fs}$ duration. The reproducibility of the quasimonoenergetic electron beam in the present experiment at a plasma density of $\simeq 8.5 \pm 0.5 \times 10^{19} \mathrm{~cm}^{-3}$ was $\sim 20 \%$. No significant improvement in the reproducibility of the quasimonoenergetic electron beam with the positive chirp was observed.

The observation of low divergence quasimonoenergetic electron beam indicates electron acceleration in highly nonlinear plasma bubble, although the initial laser and plasma density parameters in the present experiment did not satisfy the conditions necessary for such acceleration $[9,10]$. For the plasma density $n_{e} \geq 5 \times 10^{19} \mathrm{~cm}^{-3}$ investigated in the present experiment, the plasma wavelength $\lambda_{p} \leq 5 \mu \mathrm{m}$ and consequently the laser pulse length $(c \tau)$ was about 3 times or higher (for $\tau \geq 45$ fs) compared to the plasma wavelength $\lambda_{p}$. In addition, the laser power is higher than the critical power for self-focusing for the

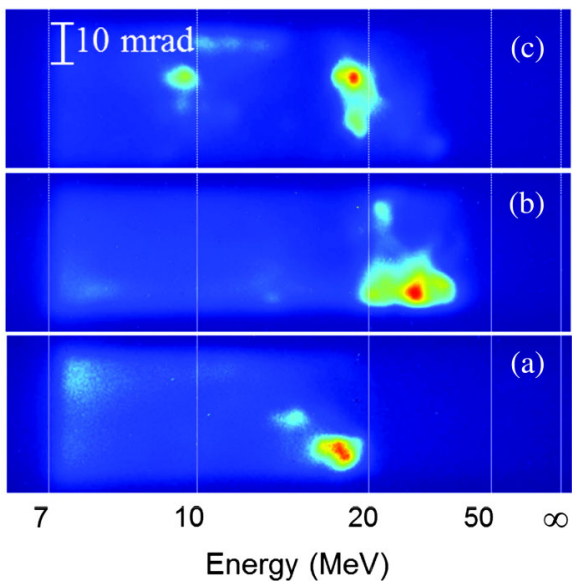

FIG. 5. Images of quasimonoenergetic electron spectra recorded at $n_{e} \simeq 8.5 \pm 0.5 \times 10^{19} \mathrm{~cm}^{-3}$, for different separations of the laser pulse compressor gratings: (a) $0 \mu \mathrm{m}$ (45 fs), (b) $-320 \mu \mathrm{m}$ (70 fs), and (c) $-640 \mu \mathrm{m}$ (135 fs). 
entire range of the laser and plasma parameters used in the experiment. These conditions are favorable for selfmodulation of the laser pulse through stimulated FRS instability [19,31]. The experimental observations also confirm the laser wakefield excitation through strong self-modulation of the laser pulse. The strong modulation of laser pulse at high plasma density in the experiment may result in breakup of initially long laser pulse into pulselets each with duration $\sim \lambda_{p} / c$ which can drive the wakefields in the bubble regime resulting in generation of quasimonoenergetic electron beam, as observed in the present experiment and also reported in previous experiments [16-18]. In the standard description of FRS, the growth of laser intensity modulations is caused by local enhancement of axial energy transport (group velocity dispersion) due to plasma wave density perturbations [31]. A linear chirp will be affected by the group velocity dispersion (GVD) in the plasma, which can elongate (for negative chirp) or compress (for positive chirp) the laser pulse, leading to rapid growth, higher accelerating fields, and a more rapid onset of particle trapping. The change in group velocity $\left(\nu_{g}\right)$ due to the frequency chirp in practical parameters is $\Delta \nu_{g} \simeq-c\left(n_{e} / n_{c}\right)(\Delta \lambda / \lambda)$ [23]. The propagation distance $L_{\mathrm{GVD}}$ required for the laser pulse to modulate at the plasma wavelength (which will drive or suppress the FRS growth) due to a linear chirp over a plasma wavelength is $L_{\mathrm{GVD}} \simeq c \lambda_{p} / \Delta \nu_{g}$. For the plasma density $n_{e} \simeq$ $6.5 \times 10^{19} \mathrm{~cm}^{-3}$ and laser parameters $\lambda=800 \mathrm{~nm}$ and $\Delta \lambda / \lambda=2.5 \%$, the propagation distance for GVD to enhance or suppress the FRS growth is $4.3 \mathrm{~mm}(2.8 \mathrm{~mm}$ at $8.5 \times 10^{19} \mathrm{~cm}^{-3}$ ), which is much greater than the growth length for the FRS instability, given by $k_{p} L_{\mathrm{GVD}} \gg$ $w_{p} / \gamma_{\mathrm{FRS}}=\left[8\left(n_{c} / n_{e}\right)\right]^{1 / 2} / a_{0}$, where $\gamma_{\mathrm{FRS}}$ is the growth rate of FRS. This indicates that, although the chirp affects the GVD throughout the pulse, the enhanced modulation due to GVD has an insignificant effect on the FRS process.

Both analytical and PIC simulations have shown that a few percent chirp (as in the present experiment) leads to a change in growth of FRS on the order of a few percent only [23]. However, in addition to the laser pulse duration, the variation in compressor grating separation also affects the pulse shape. Leemans and his co-workers [28,32] have studied the effect of compressor grating separation on laser pulse shape. They have shown that an incomplete compensation (by the pulse compressor) of the positively biased nonlinear spectral phases, which arise due to laser pulse propagation in material medium (e.g. the Ti:sapphire crystal), gives rise to pulse shape asymmetry resulting in fast/ slow rising edge when slight positive/negative chirp is introduced. This was further confirmed in a recent experiment by Hafz et al. [29] using SPIDER setup. The temporal intensity profiles of such pulses can be well fitted to a skewed Gaussian of the form $I(t)=I_{0}\left\{\left[-t^{2} /\left(2 \tau^{2}\right)\right] \times\right.$ $\left.\left[1+s t /\left(t^{2}+\tau^{2}\right)^{1 / 2}\right]^{-1}\right\}$, where $-1<s<1$ is the skew parameter which is positive (negative) for fast (slow) rise time and $\tau$ is the pulse duration, and $I_{0}$ is the peak intensity. The simulations in the self-modulated LWFA regime [28] indicate that a larger wakefield is excited for positive skewed laser pulses, compared to the negative skewed laser pulses. FRS is seeded by the density perturbations which contain frequency components at the plasma frequency. It was observed in the above simulations that the maximum amplitude of the seed decreases with increasing density or pulse duration. This explains less enhancement in charge $(25 \%)$ at $n_{e} \simeq 8.5 \pm 0.5 \times 10^{19} \mathrm{~cm}^{-3}$ compared to that $(100 \%)$ in the case of smaller $n_{e} \simeq 6.5 \pm 0.5 \times 10^{19} \mathrm{~cm}^{-3}$ observed in our experiment. For slow rise times in the case of negatively chirped laser pulse, the response is weaker and lower amplitude plasma waves ensue. A previous study [28] in the self-modulated LWFA regime has reported enhancement of electron yield by using laser pulse of intensity $>10^{19} \mathrm{~W} / \mathrm{cm}^{2}$ interacting with plasma density $n_{e} \simeq 3 \times 10^{19} \mathrm{~cm}^{-3}$. They found that the effect of envelope asymmetry on enhancement of FRS and self-modulation to be more important than that of linear chirp in pulses with symmetric temporal envelopes. The asymmetric dependence of the total electron beam charge on the grating separation observed in the present experiment agrees qualitatively well with that observed in Ref. [28], although the two experiments differ in laser intensity and plasma density. This similarity indicates qualitatively similar laser pulse shape variation with grating separation in the two experiments.

\section{CONCLUSION}

We have studied the effect of positively/negatively chirped Ti:sapphire laser pulses with duration $\geq 45 \mathrm{fs}$ and intensity $\leq 1.2 \times 10^{18} \mathrm{~W} / \mathrm{cm}^{2}$ interacting with high density $\left(>5 \times 10^{19} \mathrm{~cm}^{-3}\right)$ under dense plasma in the regime $c \boldsymbol{\tau}>d_{0} \geqslant \lambda_{p}$ for generation of relativistic electron beam. Asymmetry in the FRS signal and electron beam charge wrt the chirp was observed, with larger asymmetry occurring for lower plasma density. Enhanced FRS and nearly twofold enhancement in the accelerated electron charge could be produced with positive chirped laser pulses of $70-80$ fs duration. At sufficiently high plasma density suitable for self-modulation, low divergence quasimonoenergetic electron beam could be produced due to strong self-modulation of the laser pulse leading to acceleration in the self-modulated bubble regime. The observations have been explained on the basis of pulse shape asymmetry (fast rise time for positively chirped pulse) which arises due to uncompensated higher order phase nonlinearity in the pulse compressor. The fast rise time pulse could produce stronger seed which enhances the growth rate of the instability and the laser wakefield. Although the SM-LWFA relies on instability, the plasma wave amplitude can be controlled by controlling the seed. Since the ponderomotively excited wake dominates other noise sources in this parameter regime, pulse shaping 
provides a handle to control the seed for the instability. This offers a method for minimizing shot-to-shot output fluctuations in the beam properties in the SM-LWFA.

\section{ACKNOWLEDGMENTS}

The authors acknowledge the technical support by S. R. Kumbhare in setting up the optics and the gas jet, the laser support by R. A. Khan and R. K. Bhat, and mechanical support by R.P. Kushwaha and S. Sebastin during the experiment.

[1] T. Tajima and J. M. Dawson, Phys. Rev. Lett. 43, 267 (1979).

[2] E. Esarey, C. B. Schroeder, and W. P. Leemans, Rev. Mod. Phys. 81, 1229 (2009).

[3] S.P.D. Mangles, C.D. Murphy, Z. Najmudin, A. G. R. Thomas, J. L. Collier, A.E. Dangor, E. J. Divall, P.S. Foster, J. G. Gallacher, C. J. Hooker, D. A. Jaroszynski, A. J. Langley, W. B. Mori, P. A. Norreys, F. S. Tsung, R. Viskup, B. R. Walton, and K. Krushelnick, Nature (London) 431, 535 (2004).

[4] C. G. R. Geddes, C. Toth, J. van Tilborg, E. Esarey, C. B. Schroeder, D. Bruhwiler, C. Nieter, J. Cary, and W. P. Leemans, Nature (London) 431, 538 (2004).

[5] J. Faure, Y. Glinec, A. Pukhov, S. Kiselev, S. Gordienko, E. Lefebvre, J. P. Rousseau, F. Burgy, and V. Malka, Nature (London) 431, 541 (2004).

[6] W. P. Leemans, B. Nagler, A. J. Gonsalves, C. Toth, K. Nakamura, C. G. R. Geddes, E. Esarey, C. B. Schroeder, and S. M. Hooker, Nat. Phys. 2, 696 (2006).

[7] S. Kneip et al., Phys. Rev. Lett. 103, 035002 (2009).

[8] H. Froula et al., Phys. Rev. Lett. 103, 215006 (2009).

[9] A. Pukhov and J. Meyer-ter-Vehn, Appl. Phys. B 74, 355 (2002).

[10] W. Lu, M. Tzoufras, C. Joshi, F. S. Tsung, W. B. Mori, J. Vieira, R. A. Fonseca, and L. O. Silva, Phys. Rev. ST Accel. Beams 10, 061301 (2007).

[11] T. Y. Chien, C. L. Chang, C. H. Lee, J. Y. Lin, J. Wang, and S. Y. Chen, Phys. Rev. Lett. 94, 115003 (2005).

[12] T. Hosokai, K. Kinoshita, T. Ohkubo, A. Maekawa, M. Uesaka, A. Zhidkov, A. Yamazaki, H. Kotaki, M. Kando, K. Nakajima, S. V. Bulanov, P. Tomassini, A. Giulietti, and D. Giulietti, Phys. Rev. E 73, 036407 (2006).

[13] J. Faure, C. Rechatin, A. Norlin, A. Lifschitz, Y. Glinec, and V. Malka, Nature (London) 444, 737 (2006).
[14] A. J. Gonsalves, K. Nakamura, C. Lin, D. Panasenko, S. Shiraishi, T. Sokollik, C. Benedetti, C. B. Schroeder, C. G. R. Geedes, J. Van Tilborg, J. Osterhoff, E. Esarey, C. Toth, and W. P. Leemans, Nat. Phys. 7, 862 (2011).

[15] B. B. Pollock, C.E. Clayton, J.E. Ralph, F. Albert, A. Davidson, L. Divol, C. Filip, S. H. Glenzer, K. Herpoldt, W. Lu, K. A. Marsh, J. Meinecke, W. B. Mori, A. Pak, T. C. Rensink, J. S. Ross, J. Shaw, G. R. Tynan, C. Joshi, and D. H. Froula, Phys. Rev. Lett. 107, 045001 (2011).

[16] B. Hidding, U. K. Amthor, B. Liesfeld, H. Schwoerer, S. Karsch, M. Geissler, L. Veisz, K. Schmid, J. G. Gallacher, S. P. Jamison, D. Jaroszynski, G. Pretzler, and R. Sauerbrey, Phys. Rev. Lett. 96, 105004 (2006).

[17] B. S. Rao, A. Moorti, P. A. Naik, and P. D. Gupta, New J. Phys. 12, 045011 (2010).

[18] E. Miura, K. Koyama, S. Kato, and N. Saito, M. Adachi, Y. Kawada, T. Nakamura, M. Tanimoto, Appl. Phys. Lett. 86, 251501 (2005).

[19] W. L. Kruer, The Physics of Laser Plasma Interactions (Addison-Wesley, Redwood City, CA, 1988).

[20] G. Z. Sun, E. Ott, Y. C. Lee, and P. Guzdar, Phys. Fluids 30, 526 (1987).

[21] E. S. Dodd, and D. Umstadter, Phys. Plasmas 8, 3531 (2001).

[22] T.-W. Yau, C.-J. Hsu, H.-H. Chu, Y.-H. Chen, C.-H. Lee, J. Wang, and S.-Y. Chen, Phys. Plasmas 9, 391 (2002).

[23] C. B. Schroeder, E. Esarey, C. G. R. Geddes, Cs. Toth, B. A. Shadwick, J. van Tilborg, J. Faure, and W.P. Leemans, Phys. Plasmas 10, 2039 (2003).

[24] V. B. Pathak, J. Vieira, R. A. Fonseca, and L. O. Silva, New J. Phys. 14, 023057 (2012).

[25] S. Y. Kalmykov, A. Beck, X. Davoine, E. Lefebvre, and B. A. Shadwick, New J. Phys. 14, 033025 (2012).

[26] X. Zhang, B. Shen, L. Ji, W. Wang, J. Xu, Y. Yu, L. Yi, X. Wang, N. A. M. Hafz, and V. Kulagin, Phys. Plasmas 19, 053103 (2012).

[27] A. K. Upadhyay, S. A. Samant, and S. Krishnagopal, Phys. Plasmas 20, 013106 (2013).

[28] W. P. Leemans, P. Catravas, E. Esarey, C. G. R. Geddes, C. Toth, R. Trines, C. B. Schroeder, B. A. Shadwick, J. van Tilborg, and J. Faure, Phys. Rev. Lett. 89, 174802 (2002).

[29] N. A. M. Hafz, T. J. Yu, S. K. Lee, T. M. Jeong, J. H. Sung, and J. Lee, Appl. Phys. Express 3, 076401 (2010).

[30] C. D. Murphy et al., Phys. Plasmas 13, 033108 (2006).

[31] W. B. Mori, IEEE J. Quantum Electron. 33, 1942 (1997).

[32] Cs. Tóth, J. Faure, J. van Tilborg, C. G. R. Geddes, C. B. Schroeder, E. Esarey, and W. P. Leemans, Opt. Lett. 28, 1823 (2003). 